\title{
Transitando lo cotidiano: método autobiográfico en estudios de género en Bucaramanga, Colombia
}

\author{
Transiting the everyday: Autobiographical method \\ in gender studies in Bucaramanga, Colombia
}

\author{
Priscyll AnCtil Avoine*
}

El artículo se sitúa en una corpo-geografía poco explorada con relación a los estudios de género y las pedagogías feministas alternativas: el departamento de Santander en Colombia, en el noreste del país. Es el resultado de la aplicación empírica de un método autobiográfico creado con la intención de reflexionar sobre las posibilidades de decolonizar el aprendizaje en el contexto de cursos universitarios sobre géneros. Su objetivo es explorar la aplicación de este método en dos universidades colombianas, una privada y otra pública, a partir de notas de observación docente, así como de las interacciones que se crearon entre la profesora y las personas estudiantes que permiten pensar la teoría feminista basada en la realidad corporal, la subjetividad y la sexualidad. Anclado en las pedagogías feministas decoloniales, el artículo aborda, de manera autorreflexiva, la relevancia y las posibilidades que ofrece el método autobiográfico en estudios de género.

The present article is situated in an unexplored embodied geography with relation to Gender Studies and Alternative Feminist Pedagogies: the department of Santander in Colombia, located in the north eastern part of the country. It presents the results of empirical application of the autobiographical method created with the aim of considering possibilities to decolonize learning processes in university gender courses. As such, the purpose of this article is to explore the implementation of this method in two Colombian universities, one private and the other public, based on observations notes as well as on the interactions between the students and the teacher, suggesting that Feminist Theory can be thought from embodied reality, subjectivity and sexualities. Anchored in Decolonial Feminist Pedagogies, the article finally proposes, in a self-reflexive way, to explore the relevance and the possibilities offered by the autobiographical method in Gender Studies.

\author{
Keywords: \\ gender, \\ autobiographical \\ method, \\ Colombia, \\ decolonial \\ deminist \\ pedagogies
}

Recibido: 7 de septiembre de 2018. Aceptado para su publicación: 29 de marzo de 2019. Recuperado de: https://sinectica.iteso.mx/index.php/SINECTICA/article/view/916

DOI: 10.31391/S2007-7033(2019)0052-006

\footnotetext{
Doctoranda en Ciencias Políticas y Estudios Feministas por la Université de Québec à Montréal (Canadá). Es becaria Vanier. Participa en la ONG Corporación Descontamina en Colombia y es miembro de la Red de Estudios Latinoamericanos de Montreal. Líneas de investigación: teoría de la descolonización y reintegración de mujeres excombatientes. priscyll.anctil@gmail.com/
} http://orcid.org/0000-0003-3622-3428 


\section{INTRODUCCIÓN}

l enseñar a nivel universitario en Colombia, aprendimos mucho más de lo que A enseñamos: este texto, por lo tanto, se dibuja como una perspectiva autorreLlexiva y autobiográfica sobre nuestra experiencia en la docencia en grupos de estudiantes heterogéneos de varias disciplinas. Esto nos permitió indagar sobre el impacto del trabajo pedagógico a partir de historias de vida; como fue para los estudiantes, constituyó también una oportunidad para repensar nuestro cuerpo y sexualidad. Esta postura es la que adoptamos en este artículo: reflexionar sobre las relaciones intersubjetivas y el ejercicio de la enseñanza en un contexto difícil para la diversidad sexual como es el caso de Colombia (CIDH, 2015; Colombia Diversa, 2014).

La decisión de escribir esta experiencia surge del sentimiento que, en una Colombia marcada por la violencia, muy pocas historias son contadas, muchas veces reprimidas por la normalización de la violencia en la vida cotidiana (CNMH, 2013). En este marco, el artículo se ubica en una corpo-geografía poco explorada con relación a los estudios de género: el departamento de Santander, al nororiente de Colombia, cuya ciudad principal es Bucaramanga. Es el resultado de la aplicación empírica de un método autobiográfico creado con la intención de reflexionar sobre las posibilidades de decolonizar el aprendizaje en el contexto de los cursos universitarios de género.

El objetivo es explorar la aplicación de este método en dos universidades colombianas, una privada y otra pública, con base en las observaciones derivadas de las actividades pedagógicas realizadas en clase, así como de las interacciones entre la profesora y el grupo de estudiantes; esto, con el propósito de permitir que la teoría feminista sea pensada a partir de la realidad corporal, subjetiva y sexual.

Aquí no buscamos establecer una diferencia entre la universidad privada y la pública; de hecho, nuestras notas de práctica docente muestran que esa diferencia es mínima en el aprendizaje acerca de los estudios de género. En cada una de las instituciones, los alumnos y las alumnas confrontan situaciones y cuestionamientos similares acerca de las interrelaciones de género. Sin embargo, podemos anotar que, en Colombia, las universidades privadas suelen tener matrículas más altas que las públicas, las cuales son, en general, más accesibles a franjas de mayor diversidad de la sociedad. En términos de estructura administrativa, el funcionamiento de las privadas y las públicas se diferencia por la fuente de financiación, las posibilidades de estabilidad laborales de sus docentes y las orientaciones ideológicas (por ejemplo, muchas de las universidades privadas son de orientación católica).

Desde el punto de vista teórico, en nuestro trabajo hacemos hincapié en las pedagogías feministas decoloniales (PFD), que se han consolidado en América Latina y han buscado hacer más complejas las interrelaciones de género construidas sobre principios desiguales dentro de la dualidad colonial-modernidad (Lugones, 2008; Escobar, 2003).

Para cumplir con el objetivo, proponemos un trayecto en cuatro etapas: una parte metodológica; una presentación teórica sobre las PFD; una sección que explora las posibilidades pedagógicas del método autobiográfico para abordar las cuestiones contemporáneas sobre género; y un análisis de nuestras observaciones sobre la relevancia del método autobiográfico y las PFD en estudios de género. 


\section{Metodología}

Este artículo sigue una metodología cualitativa que se ha construido, y aún se está construyendo, a lo largo de nuestra práctica docente. Se centra en nuestra experiencia como profesora en cursos sobre estudios de género y estudios feministas durante dos años en dos universidades colombianas. El texto se apoya en nuestras notas personales acerca de las actividades prácticas realizadas por el estudiantado durante los cuatro semestres de enseñanza, y también en actividades teóricas desarrolladas para guiar el trabajo colectivo a partir de las diferentes experiencias de vida, incluyendo la nuestra.

El objetivo no es establecer el estado de las conversaciones orales y escritas con el alumnado; al contrario, es mostrar el poder de pedagogías decoloniales y del método autobiográfico con relación al género en los caminos de vida de ellas y ellos. Se trata de comenzar una reflexión sobre las dinámicas que se instalan cuando la profesora y el estudiantado están interactuando en temas complejos como las corporalidades, el género y las sexualidades.

Por lo tanto, el método autobiográfico orientó no solo las actividades educativas, sino también nuestra propia autorreflexión sobre ellas, la cual se consignó en las notas sistematizadas en este escrito. Este método se apoya en la vida cotidiana del estudiantado para convertir sus narraciones en problemas teóricos sobre las relaciones sociales de sexo. Esto también viene a unirse a los postulados de la educación popular feminista que influyó en las PFD, a saber, un cuestionamiento de las prácticas sociales cotidianas con la finalidad de transformarlas. Según Desmarais y Simon (2007), el enfoque autobiográfico, en sus dimensiones colectiva e individual, tiene tres características:

... es, ante todo una narración, hecha por un sujeto-actor, tanto oralmente como por escrito. La narración se relaciona con su vida o con una parte de ella y se sitúa en la temporalidad biográfica del sujeto-actor, articulada con la de las y los demás y con referencias históricas colectivas. Finalmente, esta narración da lugar a una búsqueda de sentido por parte del sujeto narrador (p. 356).

La teoría se presenta, entonces, "más cerca" y da lugar a la creación de contenido y marcos teóricos que servirán al estudiantado en su vida cotidiana. Permite comprender las necesidades teóricas y prácticas en cada grupo y, luego, dar cuenta de los problemas culturales desde los cuales se originan los estereotipos de género. Es, por un lado, "un enfoque de la experiencia de la formación a partir del testimonio del sujeto involucrado en dicho proceso" (De Villers, 2011, p. 35), pero también una oportunidad para que este sujeto recopile eventos disjuntos en su propia historia personal y aborde cuestiones teóricas. Finalmente, da cuenta de la "multiplicidad de ideologías presentes en el aula" y de las varias prácticas democráticas que pueden resultar (Elenes, 2001, p. 691).

Podría parecer que la autobiografía relega una vez más a "las mujeres" en la esfera privada, lo cual sería el equivalente a destacar "la ausencia de creatividad"; sin embargo, consideramos que este método puede servir como una subversión del lenguaje en estudios de género "con la condición de repensar la forma en que la vida, la escritura y el género están vinculados” (Boileau, 2008). Por lo tanto, aunque el método autobiográfico no es estrictamente hablando feminista, se manifiesta por un compromiso de la persona autora con su espacio colectivo en una temporalidad

Anctil. Transitando lo cotidiano: método autobiográfico en estudios de género en Bucaramanga, Colombia Sinéctica 52 www.sinectica.iteso.mx 
determinada, lo que permite el cuestionamiento de las relaciones de género. Este método toma diversas formas, desde el dibujo hasta el diario personal, y propicia una mediación entre lo que la persona considera público y lo privado, al atreverse a ingresar a las áreas de lo indecible (Arfuch, 2002).

\section{Contexto y desarrollo de las actividades educativas}

Desde un punto de vista institucional, las dos universidades están ubicadas en Bucaramanga, donde se asientan varias universidades públicas y privadas. Los tres cursos que hemos dictado: Género, Cuestiones de género e Introducción a las filosofías feministas, no necesariamente estaban dirigidos a estudiantes provenientes de ciencias sociales, sino también a los de sectores tan diversos como ingeniería, arquitectura, trabajo social, filosofía, odontología, optometría y derecho.

En este artículo nos centramos en las observaciones de cinco grupos en un periodo de dos años, identificados como G1, G2, G3, G4 y G5. Nuestro argumento principal destaca que el contexto cultural es más fuerte que las diferencias entre las instituciones públicas y privadas en la construcción de las relaciones sociales de sexo. De igual forma, es preciso mencionar que existen algunas diferencias respecto al contexto institucional e ideológico entre las instituciones privadas y públicas: por ejemplo, las carreras ofrecidas, el contexto ideológico, la introducción de la religión en la enseñanza o la financiación de la investigación. Sin embargo, si bien estos factores pueden influenciar ciertas visiones sobre la interrelaciones de género, nos enfocamos más en las subjetividades vividas por las personas de nuestro estudio que en las estructuras institucionales.

El itinerario metodológico se divide en tres momentos: la revisión de la literatura sobre las PFD en América Latina y el método autobiográfico; la sistematización de las notas tomadas en el diario personal sobre las actividades educativas llevadas a cabo con el estudiantado; y el análisis de nuestras observaciones a la luz de las aportaciones de las PFD.

En estos cinco grupos, el primer curso del semestre fue dedicado a la escritura de los relatos autobiográficos del estudiantado, que contó sus vivencias corporales, sexuales y de género. La dificultad de la autoevaluación, la autodescripción y la autorreflexión fueron evidentes, sin distinción de los cursos y las universidades. Entonces, para estimular las reflexiones, propusimos una serie de preguntas a las que nosotros mismos tratamos de responder durante los cursos teóricos:

-Escribe tu historia de vida sobre sexualidad y género, sobre tu relación con tu familia, tus amigos, tu entorno...

¿Cómo te percibes en tus interrelaciones de género?

¿Qué puedes decir sobre tu orientación sexual, tu identidad de género, tu percepción del feminismo o los roles de género en Colombia?

¿ ¿Vives, has vivido o conoces personas que han vivido o experimentado cambios o reflexiones personales sobre su identidad de género?

¿Cómo te construiste como hombre/mujer/otro?

¿Cómo viviste tu sexualidad? ¿Cuáles son tus sentimientos como hombre, mujer u otro género? 
La lectura cuidadosa de las historias personales del estudiantado provocó un cuestionamiento importante en nuestra práctica docente: al mismo tiempo que les planteábamos las preguntas, también nos enfrentábamos a nuestra propia subjetividad al exponer nuestras experiencias personales; este fue el inicio de un tejer de experiencias sobre las cuales construiremos esta reflexión inicial.

En este contexto, resultó importante considerar estos desafíos, por lo cual hemos recopilado nuestras impresiones y las registramos en un diario de docencia para pensar en el plan de curso a partir de las vivencias del estudiantado en el "currículo oculto", que incluye todo el sistema de valores y el aprendizaje que no hacen parte explícita del plan de estudios (Torres, 1998; Maceira, 2005). Así, el currículo oculto comprende las interacciones entre las alumnas y los alumnos y el profesorado, los valores que se construyen en estas interacciones, lo "no dicho" o las significaciones no afirmadas de manera pública. Como Maceira (2005) lo expone, este currículo oculto es la fuente de muchas prácticas sexistas en las universidades.

Al final del semestre, solicitamos la entrega del trabajo final en el formato de un portafolio, que tomó la forma artística y creativa deseada por el alumnado y que consistía en indagar sobre cinco teorías que contribuyeron a la práctica cotidiana de la igualdad de género. Ahí, ellas y ellos confrontaron sus propios prejuicios, sentimientos y experiencias compilados al principio del semestre en la autobiografía, y subrayaron el camino teórico realizado durante el semestre. Los resultados fueron tan diversos como impresionantes, al demostrar que la experiencia cotidiana podría sentar las bases para nuevas acciones comunitarias y praxis de liberación (Korol, 2009; Dussel, 1998).

De este modo, podemos resumir de la siguiente forma las etapas de recopilación de las reflexiones aquí presentadas: en cada grupo-clase, organizamos varias actividades pedagógicas derivadas de las primeras cavilaciones del estudiantado en su relato autobiográfico, a partir de lo cual trabajamos temáticas que interpelaban al grupo. Luego, recopilamos esas experiencias teóricas y prácticas, y anotamos, semanalmente, los aspectos abordados y desarrollados, además de los problemas identificados acerca de las interrelaciones de género. A partir de los aportes teóricos de las feministas, en especial decoloniales, a la pedagogía, el análisis de estas reflexiones se hizo basado en un ejercicio de categorización por temáticas a la luz de lo escrito en el diario cotidiano. La siguiente parte da cuenta de la importancia de la cotidianidad en el proceso reflexivo-interactivo (Lafortune et al., 2018).

\section{LA EXPERIENCIA COTIDIANA: LA CONTRIBUCIÓN DE LAS PEDAGOGÍAS FEMINISTAS DECOLONIALES}

Habitar el feminismo hoy, para mí, es habitar la contradicción. Espinosa-Miñoso et al. (2017)

El contexto de la educación superior colombiana está marcado por la situación política del país: más de sesenta años de guerra civil han contribuido a normalizar la violencia, además de buscar el silenciamiento de las diferencias (CNMH, 2013, 2015). En este sentido, hablar y posicionarse sobre la diversidad sexual no es una tarea fácil. Es crucial encontrar respuestas alternativas para abordar los problemas 
vividos por la población estudiantil, ya que varias personas sugieren que la educación superior ha marcado un importante punto de inflexión en la configuración de sus identidades de género. Las PFD latinoamericanas ofrecen evidencias para repensar los cursos sobre género a todos tipos de público y trabajar las transiciones experimentadas por las mujeres, los hombres o géneros diversos para resignificar y generar espacios de co-construcción de los saberes en el territorio del aula de clase, y también más allá de este.

Las PFD deben su tradición intelectual a varias corrientes de pensamiento; en primer lugar, autoras como Lélia Gonzalez han sentado un precedente para el feminismo latinoamericano. Influenciada por Fanon, e incluso antes de que Lugones retomara la teorización de la colonialidad del poder de Quijano (1992), Gonzalez fue una de las primeras en analizar la relación entre raza, sexo y género (Montanaro 2016; Gonzalez, 1988). En segundo, las PFD se nutren de las teorías de las feministas poscoloniales que cuestionan el análisis de la categoría de género de las corrientes dominantes del feminismo occidental, y muestran sus limitaciones en diferentes corpo-geografías de los continentes que se encuentran en la periferia del sistema mundo (Areiza, 2015; Dussel, 1994).

En tercer lugar, y de acuerdo con el legado de la teología de la liberación y de la pedagogía crítica en América Latina, la educación popular proporciona una de las bases teóricas para pensar sobre las PFD. Las pedagogías críticas latinoamericanas, múltiples y diversas, han contribuido al cuestionamiento del método educativo basado en la epistemología moderna. A partir de diversos autores y autoras, Cabaluz-Ducasse (2016) rastrea las siguientes orientaciones generales para estas pedagogías:

1) la naturaleza ética, política e ideológica de la educación, y la relevancia de la praxis político-pedagógica para la transformación social radical; 2) la identificación de factores alienantes y deshumanizantes en la cultura, por ende la educación entendida como proceso de concientización; 3) la necesidad de constituir espacios de autoeducación popular, "con" y "desde" los oprimidos y explotados; 4) la praxis dialógica como reconocimiento genuino - no instrumental ni formal- de los saberes populares subalternizados del Otro en tanto Otro; 5) la convicción de que la praxis pedagógica debe desarrollar y potenciar todas las facultades humanas, reivindicando las categorías de omnilateralidad e integralidad de la educación; 6) el reconocimiento del conflicto Norte-Sur y de los problemas del colonialismo y el eurocentrismo presentes en la pedagogía (p. 76).

Las PFD recuperan estos postulados argumentando que la opresión de género es uno de los fundamentos del colonialismo en América Latina, de ahí la necesidad de construir proyectos educativos basados en la interculturalidad crítica y antiopresiva. En este sentido, hooks (1994) retoma los principios de Freire para formular una crítica del sexismo de su propuesta, además de reiterar la necesidad de una "pedagogía comprometida" que debe valorar los saberes del estudiantado, pero que proponga también que el o la profesora se muestre dispuesta a contar sus propias experiencias. De acuerdo con lo anterior, durante los semestres, tratamos de responder las mismas preguntas que formulamos al estudiantado y utilizamos ejemplos de nuestras relaciones interpersonales para iniciar los debates teóricos.

Por último, es importante destacar las contribuciones teóricas del grupo modernidad/colonialidad, heredero de la teología de la liberación, de la teoría de la dependencia y de la pedagogía de Freire, entre otros. El grupo de colonialidad/modernidad resalta la especificidad de la teoría decolonial latinoamericana y establece una 
cierta ruptura con los estudios poscoloniales que toman sus fuentes de pensadores como Derrida, Foucault o Lacan (Escobar, 2003; Desai \& Sanya, 2016; Grosfoguel, 2010; Markowitz, 2005; Montanaro, 2016). Ofrece, desde la conceptualización de la colonialidad del poder, del ser y del saber (Quijano 1992), un proyecto decolonial "re-embodiment and relocation of thought" con el propósito de develar los límites epistemológicos del pensamiento moderno e imaginar otros sistemas de conocimiento (Desai \& Sanya 2016, p. 712). Según Mignolo (2009), esto es la "desobediencia epistémica" o la acción política que se desprende de los ideales de la modernidad para pensar desde una situación geopolítica opuesta a la colonialidad.

Lugones (2008) retoma las teorías de Quijano sobre la colonialidad del poder, el ser y el saber, y desarrolla una crítica del heterocentrismo del autor, al integrar las contribuciones de las feministas interseccionales y del Tercer Mundo en el análisis de la colonialidad. Argumenta sobre la constitución mutua de la colonialidad y la modernidad en cuanto a la categoría del género: la colonialidad del poder aludida por Quijano y la interseccionalidad de las opresiones nos llevan a entender el sistema de género moderno-colonial que ha contribuido a la instauración de la heterosexualidad obligatoria. Esto implicó reconfiguraciones del género en América Latina que persisten hasta la actualidad y que analizaremos en la última sección de este artículo; la heteronormatividad es la matriz de dominación que continúa regulando las relaciones sociales de género. Así, la tarea del feminismo decolonial comienza con el reconocimiento de la "diferencia colonial"; de ahí que se pueda articular la resistencia "infrapolítica” como praxis política diaria (Lugones, 2010, p. 753).

Con base en autoras como Gonzalez, Carneiro y Lugones, los feminismos decoloniales latinoamericanos se caracterizan por la construcción de una genealogía del pensamiento desde las márgenes y se ataca la "matriz de opresiones múltiples" a fin de recuperar las tradiciones del conocimiento en América Latina (Espinosa-Miñoso, 2014 , p. 8). Por lo tanto, es una "apuesta epistémica" que busca reconfigurar la comprensión del feminismo criticando las posturas hegemónicas que no encajan con las experiencias de mujeres, hombres y géneros diversos en América Latina (Espinosa-Miñoso, 2014; Hernández, 2001). Según Landaluze y Espel (2015), estas teorías "han desafiado las bases profundamente etnocéntricas de los feminismos occidentales, y han cuestionado su supuesta neutralidad, su carácter universalizador, y su poder de representación y de creación de identidades" (p. 36).

De este modo, las PFD tienen sus raíces teóricas y metodológicas en este cuestionamiento del feminismo hegemónico y en el deseo de ubicar la práctica feminista en un contexto antirracista que busca "radicalizar la crítica al universalismo en la producción de teoría” (Espinosa-Miñoso, 2014, p. 8). Es una oposición al saber descorporeizado y a la tendencia de crear conocimientos deslocalizados que generan una construcción identitaria negativa del otro/a (Carneiro, 2005). Las PFD invitan a revisar la forma de vivir el feminismo en diferentes geografías trabajando desde la corporeidad, la intersubjetividad y la "pluriversalidad epistemológica" (Walsh, 2007, p. 104).

Tanto las PFD como las observaciones derivadas de nuestras notas en el rol de profesora convergen en un aspecto esencial del feminismo latinoamericano: este debe ser pensado y materializado a partir de las experiencias vividas por los sujetos, a partir de la vida cotidiana (Gargallo, 2014). Esta práctica se opone a lo que dicta el "currículo", 
el cual, en Colombia y en varios países, se edifica como "el pilar epistemológico del discurso pedagógico", y se posiciona como una guía que establece los pasos a seguir para la realización de las actividades educativas (Areiza, 2015, p. 12). Por una parte, se trata de operar una conexión entre el conocimiento y la experiencia y, por otra, de comprender que el profesorado y el estudiantado encarnan el saber.

Conscientes de la dinámica de poder entre profesora y alumnado, así como entre el "Norte y el Sur", nos involucramos activamente en la puesta en duda colectiva sobre las relaciones de género, raza y clase que cada uno y cada una vivía. Las PFD ayudaron a cuestionar nuestro propio proceso educativo, así como la postura de "mujer blanca", que vive en Colombia, y facilitaron espacios sobre género frente a una diversidad de estudiantes que enfrentaban problemas irresueltos $y$, a veces, extremadamente tabús en el entorno donde nos encontrábamos. De hecho, esta postura justifica aún más la adopción de una metodología autobiográfica y del feminismo decolonial para las clases: el objetivo era poner en duda los planes de curso con la intención de no imponer una epistemología occidental en los ejercicios pedagógicos.

Conscientes de nuestra postura de privilegio, cuestionándola y buscando una forma de romper nuestros propios presupuestos en el ámbito pedagógico, nos aproximamos al paradigma otro (Mignolo, 2005) de las teorías decoloniales para deconstruir los tabús y normas sociales dominantes junto con los grupos-clases. Esto nos permitió trabajar los contenidos académicos a partir de las historias de las mujeres, los hombres y géneros diversos presentes en los grupos para resignificar y generar espacios de co-construcción de los saberes que posibilitan un compromiso ético entre el estudiantado y la profesora. Es una tarea de recuperación de estos saberes y de creación de saberes propios, más inteligibles en el cotidiano. Por lo tanto, se teoriza lo que es vivido y no el contrario. Es una transgresión de los espacios científicos tradicionales; una forma de disrupción académica basada en la corporalidad y la subjetividad (Anctil y Miranda, 2016). El punto de vista local se convierte en el lugar de la praxis feminista; es la situación geopolítica más cercana de la persona, la cual le permite teorizar a partir de sus propias márgenes. El cuerpo, las subjetividades, pero también las emociones, se convierten en ejes centrales para pensar acerca de las teorías de género; es la pedagogía como un ejercicio encarnado y emocional (Espinosa-Miñoso, Gómez, Lugones y Ochoa, 2013).

\section{EL RELATO AUTOBIOGRÁFICO: UNA HERRAMIENTA PEDAGÓGICA EN ESTUDIOS DE GÉNERO}

En esta sección mostramos que, a pesar de que los métodos autobiográficos no solo se inspiran en el feminismo, la creación de vínculos entre la vida del estudiantado y el material impartido está en el corazón de las pedagogías feministas. De hecho, según Perumal (2012), la investigación feminista quiso poner especial atención en las metodologías que cuestionan los factores emocionales, subjetivos o biográficos que se establecen entre los sujetos.

Como lo demuestra la metodología expuesta, el propósito del uso del método autobiográfico en los cursos de género era de identificar los principales problemas que, aunque parezcan individuales, son fundamentalmente colectivos. Para ello, la autobiografía permite profundizar en temas como el cuerpo, la sexualidad, el género, los 
feminismos; se hace posible movilizar las emociones de las personas en el aula de clase, lo cual desplaza nuestra atención: el o la profesora es el único "locus" de la enseñanza (Niccolini, 2016). Las subjetividades del estudiantado, así como las del profesorado, son entonces movilizadas y desafían las relaciones de poder y los modelos educativos basados en la división cuerpo/espíritu (Perumal, 2012).

Lo anterior permite establecer vínculos entre la narración de la persona y su contexto sociocultural, lo que hace posible diseñar el plan de clase basado en teorías que se pueden usar en la vida diaria de las personas en el aula. La autobiografía abre las puertas a la crítica de las normas y provoca sentimientos que desafían creencias establecidas. De acuerdo con Arfuch (2002), este método trabaja "la impronta de la subjetividad, esa notación diferencial de la persona que habilita el discurso de la (propia) experiencia" (p. 118), pero también las dimensiones corporales y colectivas inherentes a la subjetividad humana. En el caso de las clases de género, esto determina el tono para escribir desde/y sobre el cuerpo, un cuerpo considerado como una entidad social dependiente de las demás personas para su florecimiento (Butler, 2009).

Según Argüello (2014), el método autobiográfico "busca explorar las raíces de la acción humana a través de los relatos de los propios sujetos, teniendo en cuenta las asunciones histórico-culturales que generan múltiples referentes de significación personal" (p. 24). Así, durante los cursos, el objetivo era crear espacios de reflexión tanto colectiva como personal sobre las teorías de género basadas en la intimidad y lo cotidiano. Como profesora, esto significó también exponer al colectivo estudiantil los propios miedos, cuestionamientos, incertidumbres y experiencias personales.

De esta manera, la narración se convierte en un eje central en la formación de una persona, y ofrece la posibilidad de dar su testimonio subjetivo, al mismo tiempo que implica una toma de conciencia para esta persona de su propia existencia en el universo social (Pujadas, 1992). Es una forma de pensar sobre las experiencias propias con la finalidad de situarlas en un marco cultural de formación. Para Arfuch (2002), es a la vez "una cartografía de la trayectoria individual" y una búsqueda de enlaces colectivos (p. 17). Además, Sanz (2005) argumenta que este método favorece una mayor participación del sujeto, por lo que le supone relatar su propia historia mientras rastrea también la dimensión social de lo vivido.

Roberts (2002) señala que la historia de la vida permite "major social shifts" (p. 5), al volver las relaciones sociales más complejas y mostrar varios problemas que, hasta entonces, podrían ser invisibles en el aula. Esas problemáticas no aparecen, de modo necesario, en el caso de un curso sobre género que sigue un programa o un currículo preestablecido por la institución y que permanece insensible a las realidades vividas por el estudiantado a un momento histórico específico.

El método autobiográfico se encuentra con las pedagogías feministas decoloniales en varios puntos. Como lo señala Elenes (2001), las "pedagogías de fronteras/ transformativas" son una manera de deconstruir la noción de sujeto unificado y esencializado (2001). Además, este método da cuenta de los diferentes procesos de subjetivación -conscientes o inconscientes-, lo cual permite iniciar un itinerario de formación centrado en la decolonización de los saberes. Como menciona Maldonado-Torres (2013), con base en Fanon, esta decolonización implica “... desaprender 
lo impuesto y asumido y de volver a reconstituir el ser" (p. 11). A partir de un "yo" situado, el método autobiográfico y las PFD ponen en el centro de sus análisis las relaciones de poder heredadas del sistema moderno-colonial de género (Lugones 2008,2010 ) y ofrecen desaprender las estructuras patriarcales y racistas que contribuyen a su perpetuación.

En los cursos de género, las alumnas y los alumnos inician un posicionamiento corporal del “yo" y, en relación con las demás personas, tratan de cuestionar los marcos sexistas a partir de sus propias vivencias. Es una manera de identificar los problemas contemporáneos relacionados con el género y la sexualidad en Colombia sin que estos aparezcan como externos y otros: cada uno y cada una puede ser el otro o la otra. Esto permite considerar el género como una categoría analítica (Scott, 2011; Wills, 2014), y también su aplicación práctica e intersección con otros marcadores de opresión, como la clase o la raza.

\section{CUERPO, SEXUALIDADES Y SUBJETIVIDADES: TRANSITANDO EL COTIDIANO BUMANGUÉS}

El ámbito de alcance no es heroico, es el ámbito de la vida cotidiana Claudia Korol (2007, p. 59)

En Bucaramanga, los roles de género siguen marcos rígidos de definición de la feminidad y la masculinidad. Por un lado, las mujeres se posicionan como "guerreras", "mujeres verracas" o "arrechas", lo que tiende a destacar un carácter "fuerte" que sería "común" a todas las mujeres de la región (grupo G1, 2015). Por otro, la dominación masculina sigue afectando de manera desproporcionada a las mujeres, y da lugar a violencias múltiples. Es apenas reciente que los gobiernos regionales han empezado a prestar atención al tema de las relaciones de género y la igualdad de género en sus planes de desarrollo bajo la presión constante de los colectivos de mujeres, pero sigue habiendo muy poca financiación asignada a este tema.

Si bien el Departamento de Santander tiene una "política pública de mujer y equidad de géneros", la discriminación es generalizada con tasas muy altas de violencia basada en el género (Gobernación de Santander, 2015). La comunidad científica no escapa de este panorama y sigue marcada por relaciones jerárquicas y patriarcales, y deja poco espacio para las mujeres con una sobrerrepresentación de los hombres en los campos de la ciencia y la tecnología (Tillman \& Anctil, 2017). Por esta razón, es importante crear "prácticas socioeducativas que cuestionan y transforman la sociedad existente" (Cabaluz-Ducasse, 2016) y proponer pistas de interrogación propias a la experiencia latinoamericana, más locales y territorializadas que las que proponen los marcos hegemónicos del feminismo. La co-construcción de los saberes, pero también el cuestionamiento de sus estructuras de opresión, es de gran necesidad para reducir la violencia cultural en contra de las mujeres y las personas que no responden a los patrones sociales heteronormativos.

Durante los cursos se hizo evidente que los temas proporcionados por el programa no eran, necesariamente, consistentes con la realidad del estudiantado. Además, los obstáculos para el intercambio de experiencias se hacen cada vez más evidentes: el miedo a hablar, a ser juzgado/a o el temor a vivir diferentes tipos de discriminación. 
La profesora no escapa de estas dinámicas, como lo subraya hooks (1994); la persona que actúa como facilitadora de la clase debe contar sus propias historias, confrontar sus emociones y, también, aceptar el mostrar su propia vulnerabilidad exponiendo su narrativa. Según hooks (1994), un o una profesora que no aceptaría entrar en el mismo ejercicio narrativo que sus estudiantes estaría cayendo en una trampa de relaciones de poder coercitivas.

En este contexto, y según nuestras observaciones, se puede identificar una gran dificultad para hablar de los cuerpos, las prácticas sexuales y la identidad de género, la cual toma su origen en las estructuras sociales que moldean la construcción del conocimiento. Jones, O’Donnell y Todd (2016) argumentan que "understanding the genesis of both individuals and institutions means understanding the diverse material and affective processes through which bodies of all kinds are constituted and sustained" (p. 188). De ese modo, un método autobiográfico permite romper con algunas de estas estructuras de pensamiento con las personas presentes en el aula. Esta sección contiene los resultados del análisis de las notas sistematizadas en el diario docente sobre los éxitos y las limitaciones de este método de enseñanza.

En primer lugar, el método autobiográfico se presentó como una posibilidad para combatir la timidez en temas sensibles, y sirvió de punto de partida para darnos cuenta de que nadie vivía las realidades sexogenéricas en aislamiento. En los términos de Restrepo y Rojas (2010), se trata de romper la forma tradicional de adquisición del conocimiento y optar por una geopolítica del saber, marcada por un "locus de enunciación" desde el cual se produce. Esto nos permite retomar un postulado de la educación popular feminista: pensar una "metodología integrada, que comprende el cuerpo, el espíritu, el corazón y la mente [...] la vida íntima cotidiana de mujeres y hombres a la vez que sus vidas laborales y comunitarias" (Nadeau, 1996, p. 34).

Para el estudiantado, los ejercicios autobiográficos se mostraron como una novedad, una confrontación consigo mismo, que invitó a una forma de introspección. Era mucho más difícil que las estrategias de enseñanza habituales: por un lado, se enfrentaban a la autorreflexión, pero, por otro, tenían que desarrollar una reflexión sobre su entorno social desde múltiples puntos de vista. Hablar de sí mismo, en primera persona, impone muchas confrontaciones ideológicas para ciertas personas: por lo tanto, algunas de ellas se resignaron a usar la tercera persona para autodefinirse y contar sus propias experiencias sexogenéricas (grupo G2, 2015).

En segundo lugar, en todos los grupos de estudiantes discutimos la significación de la transición a la universidad como un cambio en sus concepciones de las relaciones de género; cómo esta transición había marcado una ruptura y permitió a algunas personas interrogar sus concepciones; otras personas afirmaron, en cambio, que la universidad reorientó su manera de pensar, lo cual no fue acompañado, necesariamente, del respaldo de sus pares de la universidad (grupo G1, 2015).

El análisis de las narrativas autobiográficas y, en especial, de las discusiones durante los semestres ayudó a trazar el contorno de las teorías que necesitábamos trabajar en cada grupo-clase. El entorno familiar, las instituciones -como la universidad o la iglesia- y también las relaciones emocionales están en el corazón de las historias personales y colectivas, y fueron criticadas y reinterpretadas. El sistema heterocentrado juega un papel sustancial al regular las relaciones de género: las 
reflexiones en torno a la heteronormatividad obligatoria nos permitieron, en casi cada grupo-clase, cuestionar las consecuencias raciales, sexuales y corporales del heterosexismo (Lugones, 2007). Una de las conclusiones fue que sigue siendo en extremado difícil vivir fuera de la "norma" que establece cuáles son las relaciones "sexualmente aceptables": muchas personas se identificaron con la heterosexualidad en un proceso de "negación", es decir, respondiendo a la norma negando cualquier vínculo con la homosexualidad. Por lo tanto, soy un "hombre" porque deseo a una "mujer" o soy una "mujer" porque las "mujeres" no me atraen en el aspecto sexual (grupo G5, 2016).

En las observaciones se resalta que seguía siendo difícil para los grupos llegar a una definición de la heterosexualidad, tanto para lo que se refiere al deseo como a la identidad de género. Así, la única forma de hacerlo era negar la asociación a cualquier práctica "fuera de lo común" (grupo G3, 2016). Por un lado, esto se manifiesta como un deseo de tener una "familia media" que cumpla con las normas impuestas por la sociedad: reafirmando la heterosexualidad sin llegar a una definición clara del concepto, sobre todo al inicio del semestre (grupo G4, 2016). Por otro, hemos podido abordar temas acerca de la conceptualización de la homosexualidad en la sociedad colombiana: varias historias que compartimos hicieron hincapié en los diferentes niveles de "aceptación" de las personas que se identificaban como lesbianas, homosexuales o andróginas. Para muchas personas, era posible ser "gay" si eras un "gay normal" que no rompería las "normas" del sistema heterosexual (grupo G2, 2015).

Es importante resaltar también los sentimientos de opresión que genera el sistema heteronormativo: varias discusiones y reflexiones giraron sobre la "monogamia como aburrida" o del patriarcado como un sistema con impactos corporales decisivos sobre las personas que se identifican como bisexuales (grupo G5, 2016). Las observaciones revelan que, en los discursos de equidad e inclusión, la heterosexualidad y los privilegios que se asocian a esta se mantienen sin cambiar en la vida cotidiana de las alumnas y los alumnos (Gray, Neary \& O'Sullivan, 2016). Entonces, estas reflexiones han permitido abordar la cuestión del sistema heteronormativo y poner en duda las jerarquías de lo "posible" respecto al deseo y la sexualidad, al abrir el camino para hablar de la orientación sexual (a menudo por primera vez). El análisis de estas subjetividades corporales muestra cómo el cuerpo se convierte en el lugar "where the physical, symbolic and sociological act together": también es el lugar que permite la resistencia y la transgresión de los marcos heteronormativos (Gray, Neary \& O'Sullivan, 2016, p. 256).

Así, la diversidad sexual se ha convertido en un tema transversal y las observaciones reafirman nuestras propias dificultades en encontrar estrategias para abordar la espinosa cuestión de las posibilidades corporales. Al inicio del semestre, el género fue asociado por la población estudiantil a "las mujeres" y su condición de "víctimas" del sistema machista. Entonces, el feminismo era más bien visto como una intención de "dominación" de las mujeres sobre los hombres. Estas confusiones teóricas provienen de los marcos de la educación moderna que busca producir conocimiento en vez de construirlo, y transforma la "diversidad en uniformidad y el holismo y la complejidad en fragmentación y reduccionismo" (Comins, 2003, p. 120).

Las biografías y actividades en clase mostraron el espacio limitado que las alumnas y los alumnos tienen para hablar sobre sus experiencias sexuales con personas 
del mismo género, sus cuestionamientos sobre la identidad andrógina o las posibilidades de la transexualidad. Las observaciones anotadas confluyen sobre la censura familiar, el rechazo vivido en los círculos de amigos y amigas o sobre la necesidad de moverse en instituciones "normativas", en las que se puede "esconder" o "camuflar" la identidad de género (grupos G1 y G2, 2015).

En tercer lugar, si bien el método autobiográfico permitió cuestionar las normas derivadas del "sistema colonial-moderno de género" (Lugones, 2007, 2008), las PFD, a su vez, sirvieron para contrarrestar los prejuicios a raíz de los postulados de la interseccionalidad, lo cual mostró diversas formas de vivir las opresiones (hooks, 2004). El enfoque sobre las historias de vida hizo posible un enfoque diferente para dar cuenta de la intersección de las opresiones, a saber, la oportunidad de "deshacer la dualidad sujeto-objeto" como lo menciona Anzaldúa (2012).

De hecho, varios problemas relacionados con la intersección de las opresiones surgieron en todos los grupos-clase; por ejemplo, la resistencia diaria de las madres cabeza de hogar, la violencia resultante del conflicto armado o las dinámicas de clase social y de género dentro del sistema universitario. En particular, quienes se identificaron como mujeres subrayaron episodios de violencia sexual durante su infancia y adolescencia; las mujeres percibieron que su vida cotidiana estaba marcada por distintas formas de violencia, como la violación, los embarazos no deseados o la violencia psicológica (grupos G5 y G6, 2016). Estas reflexiones nos han dado la oportunidad de trabajar sobre la violencia de género tanto en el ámbito de las relaciones de pareja como en el marco del conflicto armado, así como discutir los marcos culturales que las legitiman.

Finalmente, en las discusiones en clase quedó claro que la religión católica tiene un impacto considerable sobre lo que se espera de las mujeres y los hombres en Colombia. Como lo argumenta Elenes (2001), "transformative pedagogy is to enable students to demystify their own ideologies, whether on the left or the right" (p. 694), lo que provoca confrontaciones entre diferentes cosmovisiones que son, muchas veces, conectadas con las creencias religiosas. La religión, una institución central en el país, plantea preguntas importantes sobre la corporalidad y la sexualidad, ya sea que pertenezcan a una universidad católica privada, o no. En teoría, las universidades públicas no tienen ninguna afiliación religiosa y las universidades privadas suelen ser católicas. Sin embargo, las observaciones no muestran diferencias notables entre las dos instituciones: parece que es el contexto más amplio, cultural y religioso el que contribuye a dar forma a los ideales del relacionado con los cuerpos cualificados de "normales" y "anormales".

Lo religioso ocupa un lugar preponderante, ya sea para reafirmar las convicciones sexuales o para tomar una posición en contra de las discriminaciones experimentadas en este nivel. El contexto de los diálogos de paz de La Habana y la controversia en torno a la diversidad sexual han dificultado las conversaciones sobre las posturas religiosas en el aula de clase. La religión católica define con claridad lo que se entiende por "ser hombre" o "ser mujer"; por lo tanto, los cuerpos que no responden a estas normas suelen ser expulsados de la esfera política y social (Butler, 2009), y dejan pocos espacios para redefinir estas formas de sentirse hombre, mujer, trans-, etcétera. Los esquemas religiosos definen la sexualidad normativa o "aceptable"; así, la masturbación se presenta como un conflicto de identidad, un "hábito malo" o un 
sinónimo de soledad (grupos G2, 2015) y la virginidad como un ideal religioso para el matrimonio (grupos G4, 2016). Por el contrario, algunas reflexiones giran en torno a la reapropiación de la sexualidad; se aíslan de los marcos católicos y se oponen a la imposición de una "ley" religiosa en sus vidas (grupos G2, 2015 y G5, 2016).

En este contexto, la universidad y las relaciones de amistades que se tejen se configuran como un lugar de cuestionamiento de las instituciones religiosas: es un pasaje que provoca un cambio en la manera de amar, y se separa el acto "de amar" de toda relación con el sexo, género y raza, así como de todo parámetro religioso (grupo G4, 2016). En medio de estas visiones, algunas personas señalan que es posible conciliar la religión y las luchas de los movimientos LGBTIQ+, ya que algunas iglesias han aceptado los gais y las lesbianas en su seno (grupo G4, 2016). Asimismo, algunas discusiones se dieron sobre la libertad de elección sexual, en el sentido de que también ha sido "atribuida" por Dios; por ello, la fe no entra, necesariamente, en contradicción con las posibilidades corporales (grupo G1, 2015).

Con el método autobiográfico y la exploración de las PFD, las emociones y los afectos se convierten en potentes herramientas que hacen posible mover el conocimiento (Niccolini, 2016), así como un cambio en las relaciones de poder en el grupo. Se trata de reformular reflexiones compartidas en el grupo-clase y construir en forma colectiva el conocimiento (Martínez, 2016). Las historias inaudibles, finalmente, podían ser contadas y, sobre todo, transformadas a partir de la tríada contar, teorizar y cambiar (Anctil y Miranda, 2016).

Contar la historia del sujeto encarnado es, por lo tanto, contar la historia también de la colectividad y, por lo tanto, dar sentido a todo un sistema de conocimiento, al tiempo que significa las particularidades que el cuerpo puede vivir, sentir y expresar (Rosales, 2010). Como lo destaca Young (2015), es tarea del profesorado de ayudar al estudiantado a establecer conexiones entre lo que trabaja en los cursos de género y la cultura en la cual viven. De esta constatación, deducimos que la teoría no surge solo de las personas que teorizan el género, sino de las relaciones intersubjetivas narradas y compartidas.

\section{CONCLUSIONES PRELIMINARES}

Decolonizar los cursos de género en Colombia no es una tarea fácil, porque implica una necesaria reconfiguración de la categoría de género (Areiza, 2015) tanto desde el punto de vista práctico como analítico, de ahí que el objetivo de este artículo es mostrar la relevancia de los métodos autobiográficos y las PFD para los cursos en estudios de género en el contexto colombiano. De hecho, estos métodos de acercamiento al saber permitieron una incursión colectiva en las estructuras de pensamiento respecto al género, además de abrir un espacio a fin de apropiarse de las teorías feministas para las vivencias propias del estudiantado.

La tarea de sistematizar experiencias sobre esfuerzos de decolonización del aprendizaje está en constante construcción frente a los sistemas patriarcales, racistas y neocoloniales. Las vivencias del estudiantado están marcadas por varios sistemas de opresión que no se pueden jerarquizar y se deben analizar de manera simultánea (Pagé, 2014). Las teorías feministas decoloniales han permitido 
cuestionar los privilegios que se dan en las academias con la intención de poder actuar sobre sus efectos negativos: no se trata solo de una toma de conciencia de esos privilegios, sino de una ruptura con estos y, así, un cuestionamiento constante sobre las prácticas pedagógicas dentro y fuera del aula.

Finalmente, la autobiografía ofrece un terreno para convocar lo personal en las narrativas políticas y colectivas: es la posibilidad de comprender las relaciones de género de otra manera. Como Young (2015) lo señala, "las ideas dejan de ser distantes" (p. 11). Hablar del "yo" y situarse en las relaciones sociales de sexo sigue siendo un ejercicio difícil. Sin embargo, el método autobiográfico permite "transitar por la vida cotidiana" y replantearse cómo se construyen las teorías. Este artículo demuestra el valor de los saberes que se están edificando en lo cotidiano y sus contribuciones a la epistemología feminista, a los estudios de género y al movimiento feminista en general. Las voces que construyen estos saberes, a veces audibles y a veces por primera vez, merecen que nos comprometamos en dos ámbitos: la decolonización de las prácticas profesionales y la recolección de experiencias sobre esas prácticas con el objetivo de multiplicar sus efectos positivos.

\section{Agradecimientos}

Especialmente a Yira Miranda Montero, Kathryn Orcasita Benítez y Danielle Coenga Oliveira, por acompañarnos siempre en estas reflexiones pedagógicas.

\section{REFERENCIAS BIBLIOGRÁFICAS}

Anzaldúa, G. (2012). La conscience de la Mestiza. Vers une nouvelle conscience. Les cahiers du CEDREF, vol. 18, pp. 1-16.

Areiza González, T. S. (2015). Decolonizar el saber, un reto curricular en América Latina (tesis de maestría). Ibagué, Colombia: Universidad del Tolima.

Arfuch, L. (2002). El espacio biográfico: dilemas de la subjetividad contemporánea. Ciudad de México, México: Fondo de Cultura Económica.

Argüello Parra, A. (2014). Educación, biografía y derechos humanos: un estudio de Rodolfo Stavenhagen itinerante de alteridades. Bucaramanga, Colombia: Ediciones Universidad Santo Tomás.

Boileau, N. (2008). Un genre à part: l'autobiographie et la gynocritique. Recuperado de http://books.openedition.org/pur/30730?lang=fr

Butler, J. (2009). Marcos de guerra, las vidas lloradas. Madrid, España: Espasa Libros.

Cabaluz-Ducasse, J. F. (2016). Pedagogías críticas latinoamericanas y filosofía de la liberación: potencialidades de un diálogo teórico-político. Educación y Educadores, vol. 19, núm. 1, pp. 67-88.

Carneiro, S. (2005). A construção do outro como não-ser como fundamento do ser (tesis doctoral). Brasil: Universidade de São Paulo.

CIDH (2015). Violencia contra personas lesbianas, gay, bisexuales, trans e intersex en América. Comisión Interamericana de Derechos Humanos. Recuperado de http://www.oas.org/es/cidh/informes/pdfs/ViolenciaPersonasLGBTI.pdf 
CNMH (2015). Aniquilar la diferencia. Lesbianas, gais, bisexuales y transgeneristas en el marco del conflicto armado colombiano. Bogotá, Colombia: CNMH/ UARIV/USAID/OIM.

CNMH (2013). ¡Basta ya! Colombia: memoria de guerra y dignidad. Bogotá, Colombia: Imprenta Nacional.

Colombia Diversa (2014). Cuando el prejuicio mata: Informe de Derechos $\mathrm{Hu}$ manos de Personas LGBT en Colombia. Recuperado de http://www.colombiadiversa.org/2014/06/cuando-el-prejuicio-mata-informe-de.html

Comins Mingol, I. (2003). Del miedo a la diversidad a la ética del cuidado: una perspectiva de género. Convergencia, vol. 10, núm. 33, pp. 97-122.

Desai, K. \& Sanya, B. N. (2016). Towards decolonial praxis: Reconfiguring the human and the curriculum. Gender and Education, vol. 28, núm. 6, pp. 710724.

Desmarais, D. \& Simon, L. (2007). La démarche autobiographique et son objet: enjeux de production de connaissance et formation. Recherches qualitatives, hors-série, núm. 3, pp. 350-370.

De Villers, G. (2011). L'approche autobiographique: regards anthropologique et épistémologique, et orientations méthodologiques. Recherches sociologiques et anthropologiques, vol. 42, núm. 1. Recuperado de http://rsa.revues. org/653

Dussel, E. (1998). Ética de la liberación en la edad de la globalización y de la exclusión. Madrid, España: Editorial Trotta.

Dussel, E. (1994). 1492: el encubrimiento del otro: hacia el origen del mito de la modernidad. La Paz, Bolivia: Plural.

Elenes, A. C. (2001). Transformando fronteras: Chicana feminist transformative pedagogies. International Journal of Qualitative Studies in Education, vol. 14, núm. 5, pp. 689-702.

Escobar, A. (2003). Mundos y conocimientos de otro modo: el programa de investigación de modernidad/colonialidad latinoamericano. Tabula Rasa, vol. 1 , pp. 51-86.

Espinosa-Miñoso, Y., Anctil Avoine, P., Miranda Montero, Y.I. y Orcasita Benítez, K. (2017). Habitar el feminismo hoy, para mí, es habitar la contradicción. Revista Descontamina, vol. 4, núm. 1, pp. 6-9.

Espinosa-Miñoso, Y. (2014). Una crítica descolonial a la epistemología feminista crítica. El Cotidiano, vol. 184, pp. 7-12.

Espinosa-Miñoso, Y., Gómez, D., Lugones, M. y Ochoa, K. (2013). Reflexiones pedagógicas en torno al feminismo descolonial: una conversa en cuatro voces. En C. Walsh (coord.). Pedagogías decoloniales: prácticas insurgentes de resistir, (re)existir y (re) vivir (pp. 403-443). Quito, Ecuador: Abya Yala.

Gargallo Celentani, F. (2014). Feminismos desde Abya Yala: ideas y proposiciones de las mujeres de 607 pueblos en nuestra América. Ciudad de México, México: Editorial Corte y Confección.

Gobernación de Santander (2015). Política pública de mujer y equidad de género en Santander: Plan decenal de igualdad de oportunidades de Santander. Recuperado de http://historico.equidadmujer.gov.co/mecanismos/Documents/ santander/Gobernaci\%C3\%B3n\%20de\%20Santander/Politica-publica-mujer-equidad-genero-santander.pdf 
Gonzalez, L. (1988). Por um feminismo afrolatinoamericano. Revista Isis Internacional, vol. 9, pp. 133-141.

Gray, B., Neary, A. \& O'Sullivan, M. (2016). A queer politics of emotion: Reimagining sexualities and schooling. Gender and Education, vol. 28, núm. 2, pp. 250-265.

Grosfoguel, R. (2010). Vers une décolonisation des «uni-versalismes» occidentaux : le «pluriversalisme décolonial» d’Aimé Césaire aux zapatistes. En F. Vergès et al. (coord.). Ruptures postcoloniales: les nouveaux visages de la société française (pp.119-138). París, Francia: La Découverte.

Hernández Castillo, A. (2001). Entre el etnocentrismo feminista y el esencialismo étnico: las mujeres indígenas y sus demandas de género. Debate Feminista, vol. 24, núm. 12, pp. 206-229.

hooks, b. (2004). Mujeres negras. Dar forma a la teoría feminista. En b. hooks et al. (coord.). Otras inapropiables: feminismos desde las fronteras (pp. 33-50). Madrid, España: Traficantes de Sueño.

hooks, b. (1994). Teaching to transgress: Education as the practice of freedom. Nueva York, Estados Unidos: Routledge.

Jones, R., O’Donnell, A. y Todd, S. (2016). Shifting education's philosophical imaginaries: Relations, affects, bodies, materialities. Gender and Education, vol. 28, núm. 2, pp. 187-194.

Korol, C. (2009). Hacia una pedagogía feminista. Pasión y política en la vida cotidiana. Colectiva feminista La Revuelta, recuperado de http://larevuelta. com.ar/2009/06/25/hacia-una-pedagogia-feminista-pasion-y-politica-enla-vida-cotidiana/, consultado el 25 de agosto de 2018.

Korol, C. (coord.) (2007). Hacia una pedagogía feminista: géneros y educación popular. Buenos Aires, Argentina: Editorial El Colectivo.

Lafortune, L., Gervais, L., Lacharité, B., Maheu, J., St-Cerny, A., Guberman, N., Coenga-Oliveira, D. y Anctil Avoine, P. (2018). La pédagogie féministe intersectionnelle socioconstructiviste de Relais-femmes dans son travail d'accompagnement-formation: des compétences à développer. Recherches féministes, vol. 31, núm. 1, pp. 45-64.

Landaluze, I. Z. y Espel, L. I. (2015). Los feminismos africanos. Las mujeres africanas 'en sus propios términos'. Relaciones Internacionales, vol. 27, pp. 33-54.

Lugones, M. (2010). Toward a decolonial feminism. Hypatia, vol. 25, núm. 4, pp. 742-759.

Lugones, M. (2008). Colonialidad y género. Tabula Rasa, vol. 9, pp. 73-101.

Lugones, M. (2007). Heterosexualism and the colonial/modern gender system. Hypatia, vol. 22, núm. 1, pp.186-209.

Maceira Ochoa, L. (2005). Investigación del currículo oculto en la educación superior: alternativa para superar en sexismo en la escuela. Revista de Estudios de Género. La Ventana, vol. 21, pp. 187-227.

Maldonado-Torres, N. (2013). A modo de comentario inicial. En C. Walsh (coord.). Pedagogías decoloniales: prácticas insurgentes de resistir, (re)existir y (re)vivir (pp.11-13). Quito, Ecuador: Abya Yala.

Markowitz, L. (2005). Unmasking moral dichotomies: Can feminist pedagogy overcome student resistance? Gender and Education, vol. 17, núm. 1, pp. 39-55. 
Martínez Martín, I. (2016). Construcción de una pedagogía feminista para una ciudadanía transformadora y contrahegemónica. Foro de Educación, vol. 14, núm. 20, pp. 129-151.

Mignolo, W. D. (2009). Desobediencia epistémica (II). Pensamiento independiente y libertad de-colonial. Otros Logos: Revista de Estudios Críticos, vol. 1, núm. 1, pp. 8-42.

Mignolo, W. D. (2005). 'Un paradigma otro': colonialidad global, pensamiento fronterizo y cosmopolitanismo crítico. Dispositio, vol. 25, núm. 52, pp. 127-146.

Montanaro Mena, A. M. (2016). Herencias genealógicas del feminismo decolonial en América Latina: hacia la construcción de un tercer feminismo (tesis de Maestría). Madrid, España: Universidad Carlos III.

Nadeau, D. (1996). Educación popular feminista: creando una nueva teoría y práctica. Palabra de Mujer, pp. 33-35. Recuperado de http://journals.lib. washington.edu/index.php/aquelarre/article/viewFile/13496/11846

Niccolini, A. (2016). Animate affects: Censorship, reckless pedagogies, and beautiful feelings. Gender and Education, vol. 28, núm. 2, pp. 230-249.

Pagé, G. (2014). Sur l'indivisibilité de la justice sociale ou Pourquoi le mouvement féministe québécois ne peut faire l'économie d'une analyse intersectionnelle. Nouvelles pratiques sociales, vol. 26, núm. 2, pp. 200-217.

Perumal, J. C. (2012). Bodies as objects of pedagogic power relations. Gender and Education, vol. 24, núm. 1, pp. 57-71.

Pujadas Muñoz, J. J. (1992). El método biográfico: el uso de las historias de vida en ciencias sociales. Madrid, España: Centro de Investigaciones Sociológicas.

Quijano, A. (1992). Colonialidad y modernidad/racionalidad. En R. Blackburn (coord.). Los conquistados. 1492 y la población indígena de las Américas (pp. 437-447). Bogotá, Colombia: Tercer Mundo Editores.

Restrepo, E. y Rojas, A. (2010). Inflexión decolonial: fuentes, conceptos y cuestionamientos. Popayán, Colombia: Editorial Universidad del Cauca.

Roberts, B. (2002). Biographical research. Philadelphia, Estados Unidos: Open University Press.

Rosales Cueva, J. H. (2010). Cuerpo y significación. Revista UIS Humanidades, vol. 38, núm. 1, pp. 27-39.

Sanz Hernández, A. (2005). El método biográfico en investigación social: potencialidades y limitaciones de las fuentes orales y los documentos personales. Asclepio, vol. LVII, núm. 1, pp. 99-115.

Scott, J. W. (2011). Género: ¿todavía una categoría útil para el análisis? La Manzana de la Discordia, vol. 6, núm. 1, pp. 95-101.

Tillman, R. \& Anctil Avoine, P. (2017). Rethinking gender and inter/transdisciplinarity in research and sciences. En D. A. Botero Urquijo et al. (eds.). Perspectivas sobre género: una mirada desde las ciencias humanas (pp. 55-70). Bucaramanga, Colombia: Editorial UNAD.

Torres Santomé, J. (1998). El curriculum oculto (6⿳a. ed.). Madrid, España: Morata.

Walsh, C. (2007). ¿Son posibles unas ciencias sociales/culturales otras? Reflexiones en torno a las epistemologías decoloniales. Nómadas, vol. 26, pp. 102-113. Recuperado de http://www.ucentral.edu.co/images/editorial/nomadas/docs/nomadas_8_son_posibles_catherine.pdf 
Wills Obregón, M. E. (2014). La categoría de género: nuevos retos y aperturas para la ciencia política. Colombia Internacional, vol. 80, pp. 8-16.

Young, T. G. (2015). Creative writing pedagogy: The autobiographical narrative in hybrid projects as a means to explore intersectionality (tesis de maestría). Eastern Illinois University, Estados Unidos. 